Article

\title{
Hedgehog Signal Inhibitor GANT61 Inhibits the Malignant Behavior of Undifferentiated Hepatocellular Carcinoma Cells by Targeting Non-Canonical GLI Signaling
}

\author{
Kensuke Harada, Ryuya Ohashi, Kyoko Naito and Keita Kanki * \\ Department of Biomedical Engineering, Faculty of Engineering, Okayama University of Science, 1-1 Ridai-cho, \\ Kita-ku, Okayama 700-0005, Japan; t15s047hk@ous.jp (K.H.); t16s011or@ous.jp (R.O.); t20mm02nk@ous.jp (K.N.) \\ * Correspondence: kkanki@bme.ous.ac.jp
}

Received: 11 March 2020; Accepted: 27 April 2020; Published: 28 April 2020

check for updates

\begin{abstract}
The Hedgehog (HH)-GLI pathway plays an important role in cell dedifferentiation and is therefore pivotally involved in the malignant transformation of cancer cells. GANT61, a selective inhibitor of GLI1 and GLI2, was reported as a promising treatment for cancer in various tissues; however, the biological impact of GANT61 in hepatocellular carcinoma (HCC), especially in undifferentiated HCC cells, remains unclear. In this study, we investigated the antitumor effect of GANT61 using two undifferentiated hepatoma cell lines: HLE and HLF. Quantitative PCR and RT-PCR analyses revealed that these cells express GLI transcripts, showing mesenchymal phenotypes characterized by the loss of epithelial and hepatic markers and specific expression of epithelial-mesenchymal transition (EMT)-related genes. GANT61 significantly reduced the proliferation and cell viability after drug treatment using 5-FU and Mitomycin C. We showed that GLI transcript levels were down-regulated by the MEK inhibitor U0126 and the Raf inhibitor sorafenib, suggesting that non-canonical signaling including the Ras-Raf-MEK-ERK pathway is involved. Sphere formation and migration were significantly decreased by GANT61 treatment, and it is suggested that the underlying molecular mechanisms are the down-regulation of stemness-related genes (Oct4, Bmi1, CD44, and ALDH) and the EMT-related gene Snail1. The data presented here showed that direct inhibition of GLI might be beneficial for the treatment of dedifferentiated HCC.
\end{abstract}

Keywords: GANT61; non-canonical Hedgehog-GLI signaling; hepatocellular carcinoma

\section{Introduction}

Hepatocellular carcinoma (HCC), the most frequently diagnosed primary liver cancer, is an aggressive disease caused by chronic hepatitis B or C virus infection, excessive alcohol intake, non-alcoholic steatohepatitis, or exposure to aflatoxin B [1]. The prognosis for patients with advanced stage HCC is poor because of its high rates of recurrence and intrahepatic metastasis. Consequently, there is an essential requirement for the development of an improved therapeutic strategy that aims to inhibit tumor progression. The malignant transformation of tumor cells is related to dedifferentiation of the cells [2]. A loss of cellular specific functions, increased proliferation, and enhanced glycolytic activity are the common features of tumor cells. Additional genetic mutations often cause aberrant activation of intracellular signaling pathways, leading to a loss of cell-cell adhesion, increased cell motility, and anchorage-independent cell proliferation, which are the crucial malignant phenotypes that trigger invasiveness and metastasis [3].

The Hedgehog $(\mathrm{HH})$ pathway, originally identified as an important pathway during embryonic development, is known to play a role in carcinogenesis in various tissues including the liver [4]. In 
recent years, inappropriate $\mathrm{HH}$ signaling was demonstrated in more than $30 \%$ of human cancers [5]. Canonical activation of $\mathrm{HH}$ signaling is triggered by the binding of $\mathrm{HH}$ ligands ( $\mathrm{SHH}, \mathrm{IHH}$, and $\mathrm{DHH}$ ) to their receptor Patched-1 (PTCH) on the surface of target cells. Ligand-bound PTCH lost its inhibitory regulation on Smoothened (SMO); thus, active SMO causes the downstream glioma-associated homologue (GLI) transcription factors GLI1 and GLI2 to be activated via nuclear translocation [6,7]. GLI target genes include factors involved in cell proliferation, survival, self-renewal, and invasiveness; therefore, aberrant activation of this signal pathway has a positive correlation with poor prognosis [8,9]. Small-molecule inhibitors of the HH-GLI signal, such as SMO inhibitors, were developed to increase the clinical benefit of patients. Some have entered clinical trials as promising therapies for advanced cancers, including basal cell carcinoma and medulloblastoma [10-12]. However, several patients were reported to be resistant to the SMO inhibitor because of an acquired mutation in SMO that disrupts drug binding and mutations in genes downstream of SMO, such as SUFU mutations [13-15]. Moreover, an alternative activation pathway of GLI, namely the non-canonical HH pathway, has been identified [16]. These problems of drug resistance led to the development of new small molecules that function as direct inhibitors of GLI transcription.

GANT61 is a selective inhibitor of GLI1- and GLI2-mediated gene transactivation. Preclinical studies were performed for numerous cancer types, including rhabdomyosarcoma, neuroblastoma, and leukemia, as well as colon, pancreas, cervix, skin, lung, head-neck, and gastric cancers [5]. Although substantial promising results were reported, the biological impact of GANT61 in HCC, in particular undifferentiated HCC cells, has not been clarified. In this study, we investigated the antitumor effect of GANT61 in undifferentiated HCC cells, which show greater expression of GLI transcription factors than well-differentiated HCC cells. The presented data show that GANT61 significantly reduced cell proliferation and viability after drug treatment. Malignant phenotypes, such as sphere formation and migration, were significantly decreased with reduced expression of stemness-related and epithelial-mesenchymal transition (EMT)-related genes. We also found that the gene expression of GLI is regulated, in part, by non-canonical signaling, including the Ras-Raf-MEK-ERK pathway, in these cells. Our data suggest that the application of a direct inhibitor of GLI transcription might be beneficial for the treatment of dedifferentiated HCC.

\section{Results}

\subsection{Preferential Expression of GLI Genes in Undifferentiated HCC Cell Lines}

To determine the intracellular status of GLI-mediated signaling in hepatoma cell lines, a gene expression analysis of GLI1, GLI2, and GLI3 was performed on a panel of hepatoma cells including three differentiated (HepG2, HuH1, and HuH7) and two undifferentiated (HLE and HLF) types of HCC cell. Quantitative PCR revealed that GLI1 mRNA is expressed in both differentiated and undifferentiated HCC cells, being highly expressed in undifferentiated cells (Figure 1a). Among HCC cells, HepG2, a typical well-differentiated type of HCC cell showed the lowest expression of the GLI1 gene. The expression of GLI2 and GLI3 was positively detected in undifferentiated cells but not in differentiated HCC cells. Morphologically, HepG2 showed an epithelial-like shape characterized by tight cell adhesion, while HLE and HLF showed mesenchymal morphology characterized by loose cell contact and an irregular cell shape (Figure 1b). RT-PCR analysis revealed that HLE and HLF lack the expression of E-cadherin and hepatic markers ( $A L B$ and HNF4 $\alpha$ ), but these cells express mesenchymal markers such as vimentin, CD44, snail1, and twist1 (Figure 1c). These data suggest that GLI-mediated signaling is activated in undifferentiated HCC cell lines showing the mesenchymal phenotype. Therefore, we chose HLE and HLF cells to investigate the effect of the HH signaling inhibitor GANT61. 
(a)

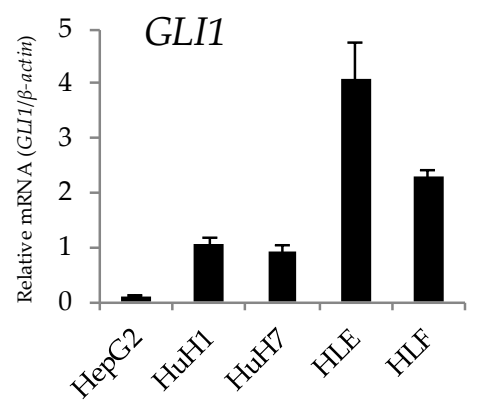

(b)

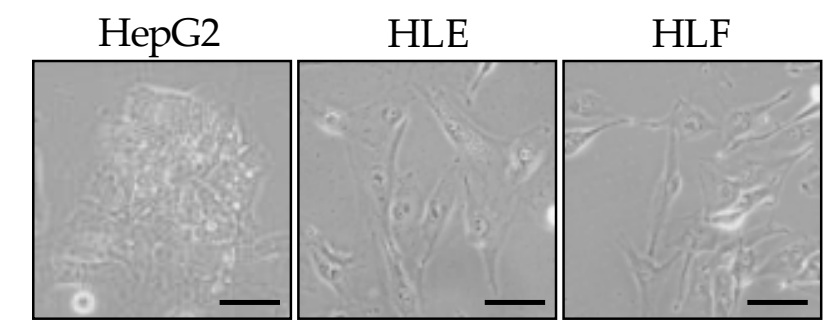

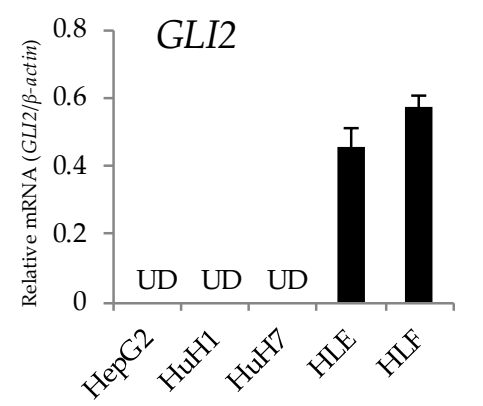

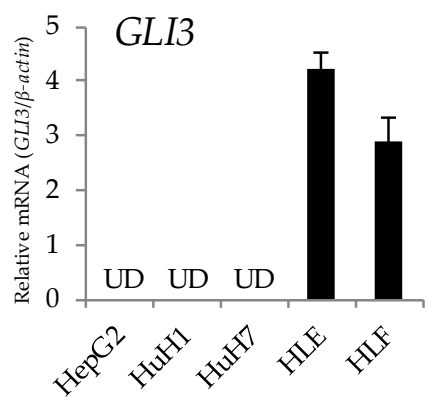

(c)

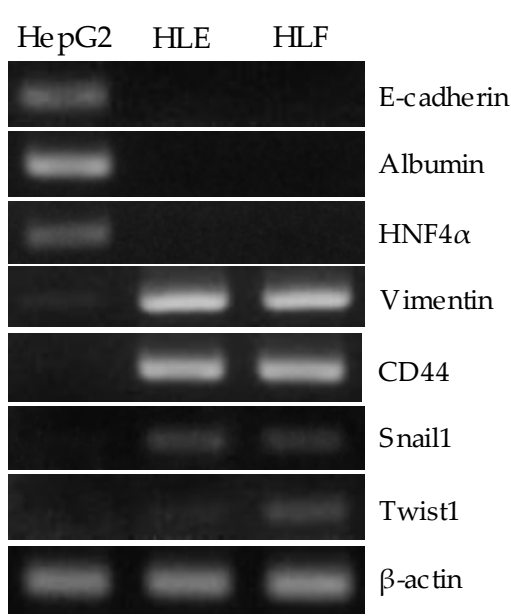

Figure 1. Preferential expression of GLI genes in undifferentiated hepatocellular carcinoma (HCC) cell lines. (a) Relative gene expression levels of GLI1, GLI2, and GLI3 compared to $\beta$-actin were determined in hepatoma cell lines by qRT-PCR analysis (UD; undetectable). (b) Microscopic observation of HepG2 (left), HLE (center), and HLF (right). Scale bar $=20 \mu \mathrm{m}$. (c) RT-PCR analysis of cell-type specific markers including E-cadherin (epithelial), Albumin, HNF4 $\alpha$ (hepatic), Vimentin, CD44, Snail1, Twist1 (mesenchymal), and $\beta$-actin as a house keeping gene.

\subsection{Antitumor Effect of GANT61 on the Proliferation of Undifferentiated HCC Cells}

To evaluate the antitumor potential of GANT61, cell proliferation was investigated in HLE and HLF cells treated with $0-10 \mu \mathrm{M}$ of GANT61. No statistically significant effect on cell proliferation was observed during the first two days of treatment (Figure 2a). In both types of cell, significant inhibition of cell proliferation was observed on day 3 of $10 \mu \mathrm{M}$ treatment (Figure 2b). Treatment with a concentration greater than $10 \mu \mathrm{M}$, for example, with 20 or $30 \mu \mathrm{M}$, markedly decreased cell viability at days 2 and 3 of the experiment (Figure 2c). Therefore, we selected a treatment with $10 \mu \mathrm{M}$ of GANT61, a quantity which did not largely affect the viability of HCC cells, for further experiments. 
(a)
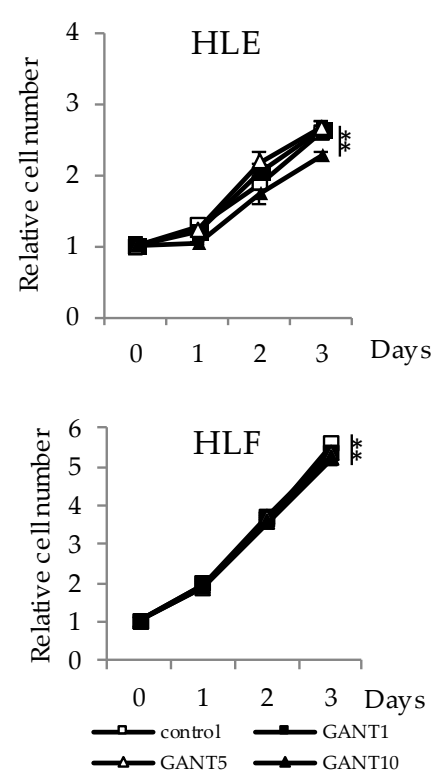

(b)
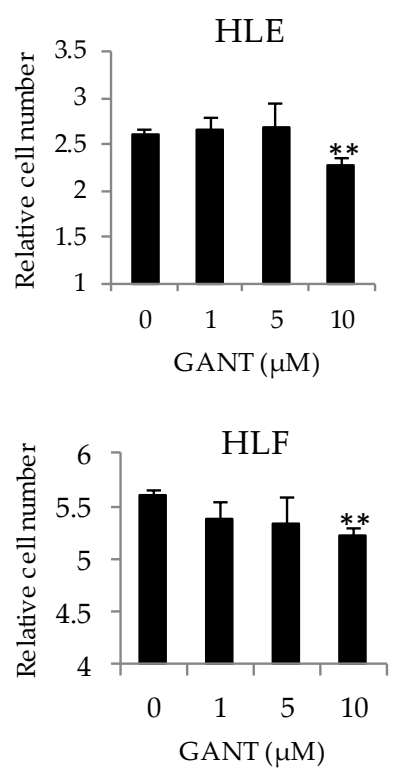

(c)
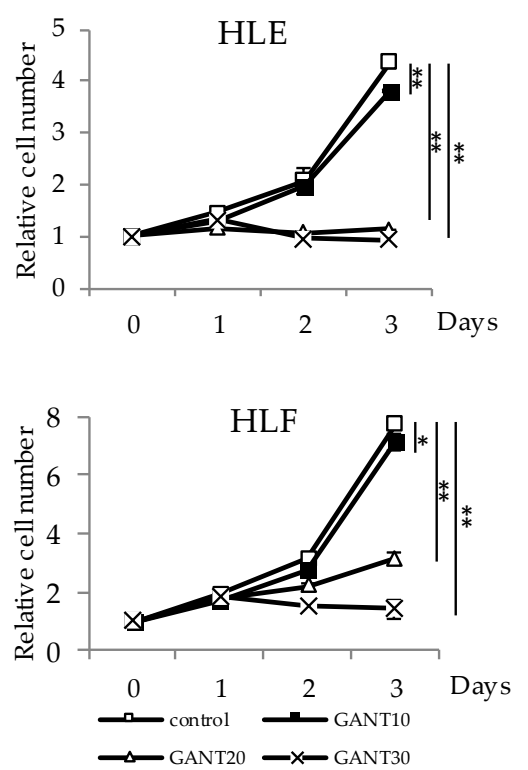

Figure 2. Antitumor effect of GANT61 on the cell proliferation of undifferentiated HCC cells. (a) HLE and HLF cells were treated with $0,1,5$, and $10 \mu \mathrm{M}$ of GANT61 for 3 days for cell proliferation assays. Cell viability was determined by WST assay and expressed as the relative number of viable cells compared to day 0. (b) Column graphs showing the values at day 3 of the experiment. (c) HLE and HLF cells were treated with $0,10,20$, and $30 \mu \mathrm{M}$ of GANT61 for 3 days and analyzed as described in (a). ${ }^{*} p<0.05,{ }^{* *} p<0.01$ vs. the vehicle control (Dimethylsulfoxide; DMSO).

2.3. Drug-Specific Enhancing Effect of Cytotoxicity by GANT61 and Involvement of the Ras-Raf-MEK-ERK Pathway in the Regulation of GLI Expression in Undifferentiated HCC Cells

Next, to examine the effect on drug sensitivity, HLE and HLF cells were treated with a series of concentrations of Mitomycin C (0-50 $\mu \mathrm{g} / \mathrm{mL}), 5-\mathrm{FU}(0-500 \mu \mathrm{g} / \mathrm{mL})$, and sorafenib $(0-50 \mu \mathrm{g} / \mathrm{mL})$ in combination with GANT61 for $48 \mathrm{~h}$. GANT61 was used at $10 \mu \mathrm{M}$ which showed no inhibitory effect on the proliferation of HLE and HLF cells up to $48 \mathrm{~h}$ after the treatment. The three anticancer drugs significantly reduced the cell viability of HLE and HLF cells in a dose-dependent manner; however, the effect of combination treatment with GANT61 differed between the drugs. GANT61 significantly reduced the viability of HCC cells treated with relatively high concentrations of mitomycin C and 5-FU. In contrast, this enhancement of drug sensitivity was barely observed in the combination with sorafenib (Figure 3a,b). To understand the mechanism of the drug-specific effect of GANT61, we next examined the expression level of GLI mRNA in sorafenib-treated cells. Sorafenib is known as a multi-kinase inhibitor that targets the kinase activity of B-Raf, vascular endothelial growth factor receptors (VEGFRs), and platelet-derived growth factor receptor $\beta$ (PDGFR- $\beta$ ). We first examined the amount of the active forms of MEK1 and ERK1/2, downstream molecules of the Ras-Raf pathway, in HLE cells treated with the inhibitors. Phosphorylated forms of MEK1 (p-MEK1) and ERK1/2 (p-ERK1/2) were shown to be decreased by treatment with the MEK inhibitor U0126 $(1 \mu \mathrm{M})$ and sorafenib $(10 \mu \mathrm{M})$ (Figure 3c,d). Quantitative PCR revealed that these treatments significantly decreased the expression levels of GLI1, GLI2, and GLI3 mRNA (Figure 3e). Altogether, these data suggest that GANT61 has an antitumor effect by decreasing cell proliferation and cell viability when it is combined with anticancer drugs in undifferentiated HCC cells. The effect of combination treatment on drug sensitivity differed between the drugs due to the non-canonical regulation of GLI-mediated signaling by the Ras-Raf-MEK-ERK pathway, a critical target of sorafenib. 
(a)

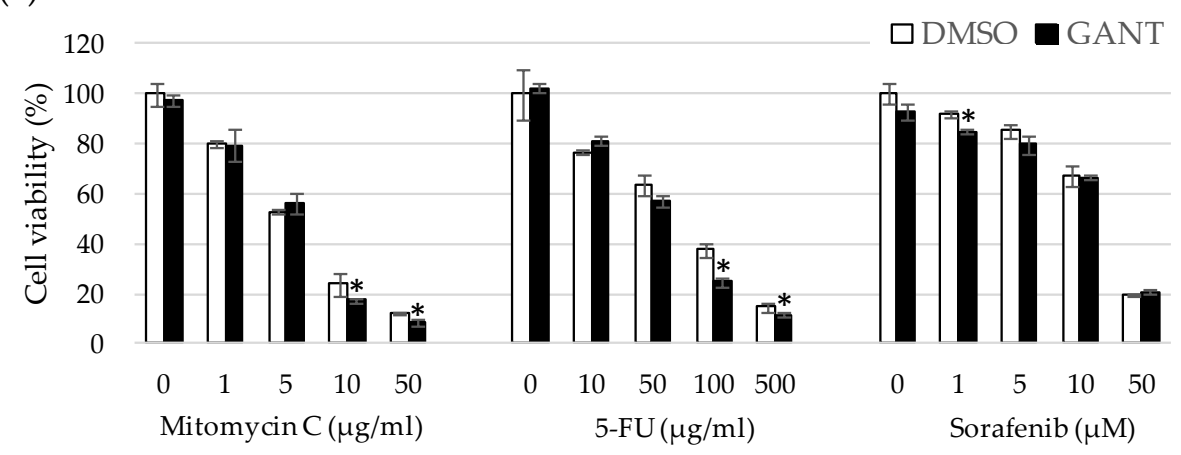

(b)

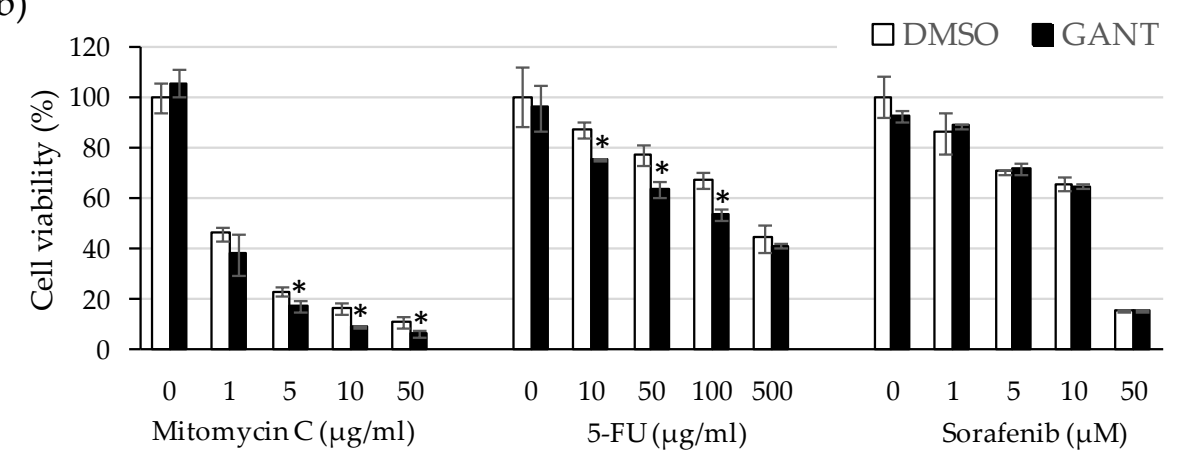

(c)

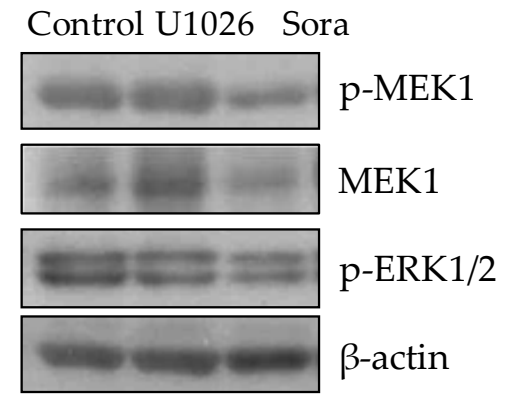

(d)

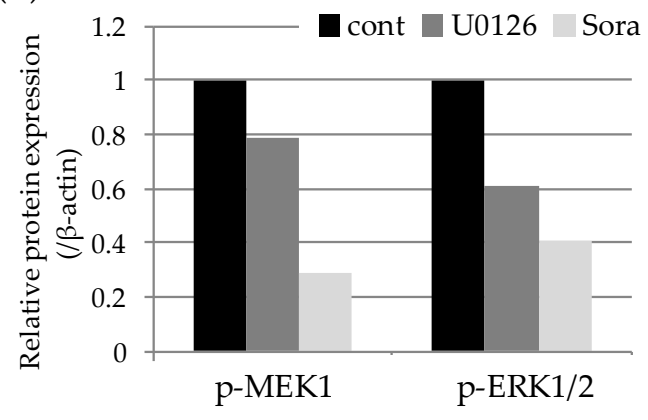

(e)

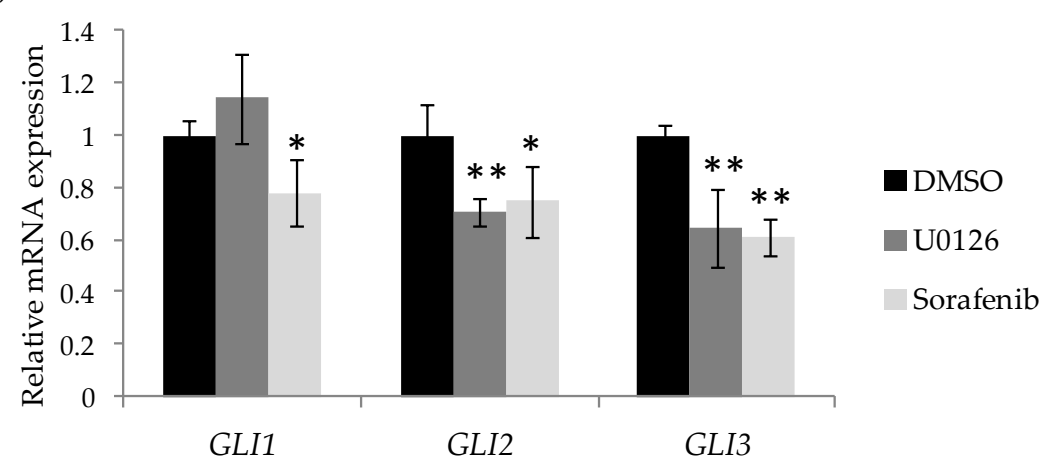

Figure 3. Drug-specific enhancing effect of cytotoxicity by GANT61 and involvement of the Ras-Raf-MEK-ERK pathway in the regulation of GLI expression in undifferentiated HCC cells. Cell viability assays of HLE (a) and HLF (b) cells treated with $10 \mu \mathrm{M}$ of GANT61 in combination with various concentrations of anticancer drugs. After $48 \mathrm{~h}$, cell viability was measured by WST assay and is expressed as the percentage of drug-free control. (c) Western blot analysis of phospho-MEK 
(Ser2017/221), total MEK, and phospho-ERK (Thr202/Tyr204) in the lysate extracted from the cells treated with U1026 $(1 \mu \mathrm{M})$ and sorafenib (Sora) $(10 \mu \mathrm{M})$ for $24 \mathrm{~h}$. $\beta$-Actin served as a control of protein loading. (d) Relative protein expression levels were calculated from the band intensity with ImageJ software (NIH). (e) Relative gene expression levels of GLI1, GLI2, and GLI3 in U1026- and sorafenib-treated cells compared to the control (DMSO). ${ }^{*} p<0.05,{ }^{* *} p<0.01$ vs. the vehicle control (DMSO).

\subsection{GANT61 Inhibits the Sphere Forming Capacity of Undifferentiated HCC Cells}

To examine the effect of GANT61 on the cancer stem cell (CSC)-like phenotype, sphere formation in suspension culture was performed in the presence or absence of GANT61. HLE and HLF cells were able to form large spheres in a low adherence plate with media containing the vehicle. In contrast, large spheres were barely observed in the presence of $10 \mu \mathrm{M}$ of GANT61 (Figure 4a,b). To understand the possible molecular mechanism underlying this, we next examined the expression of stemness-related genes in control and GANT61-treated cells. Quantitative PCR revealed that Oct4, a transcription factor related to pluripotency and self-renewal, was significantly down-regulated in HLE cells treated with GANT61. In HLF cells, GANT61 treatment showed a tendency to reduce the expression of three genes-Oct4, Bmi1 and CD44-although the difference was not statistically significant (Figure 4c). Two subtypes of aldehyde dehydrogenase, ALDH1A1 and ALDH1A3, were significantly down-regulated by GANT61 in HLF and HLE cells, respectively. These data suggest that GANT61 inhibits sphere formation by down-regulating stemness-related genes in undifferentiated HCC cells.

(a)
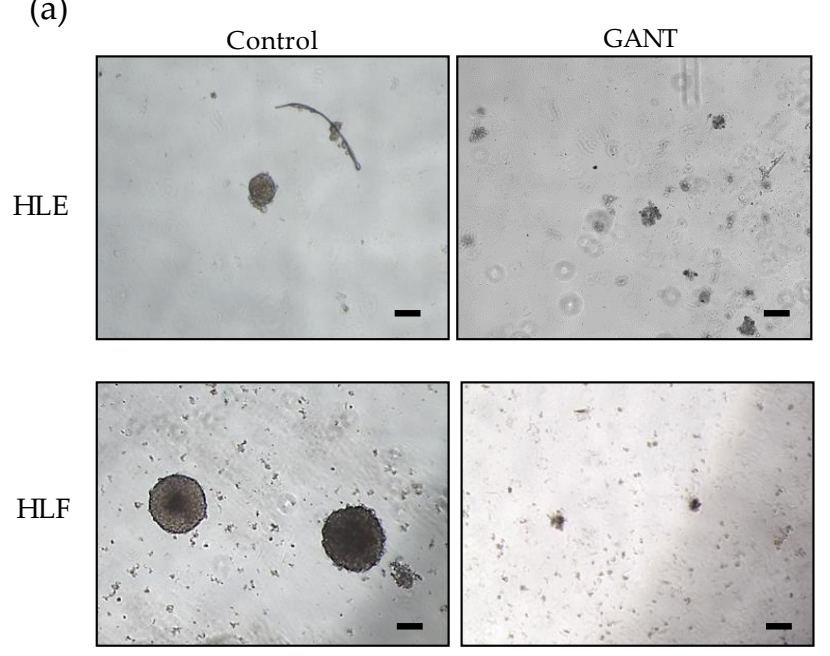

(b)
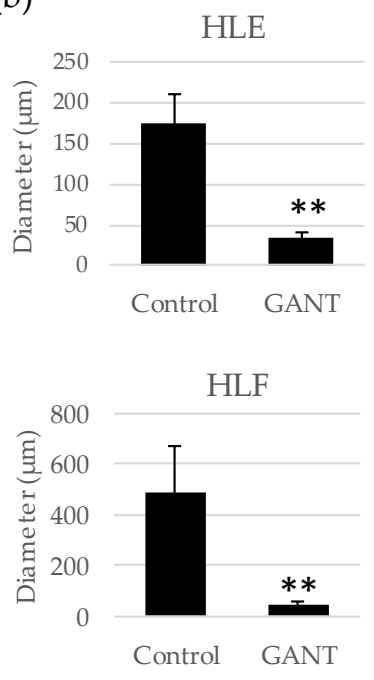

(c)
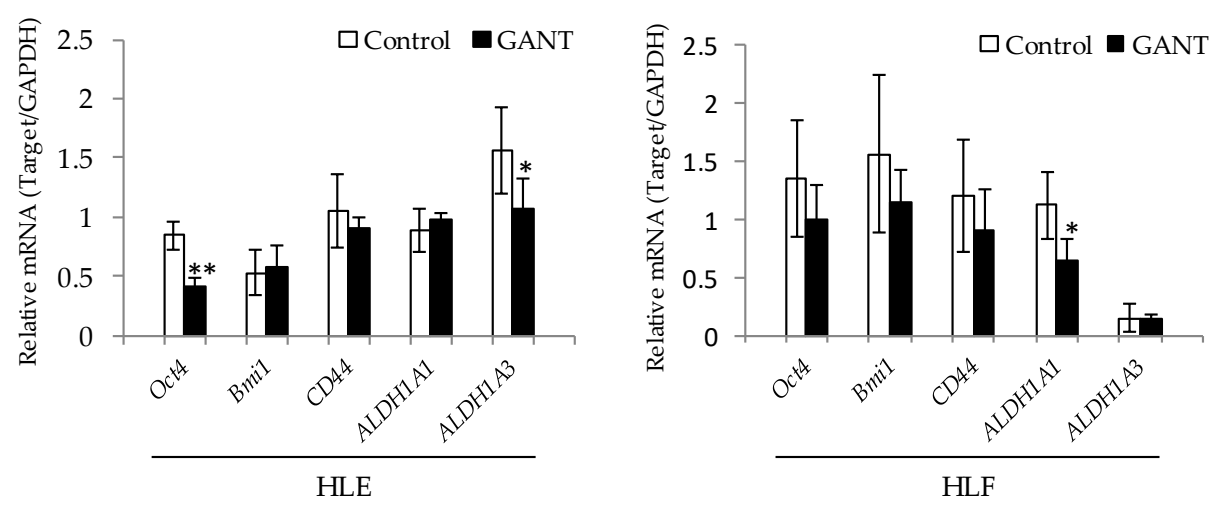

Figure 4. GANT61 inhibits sphere formation by down-regulating stemness-related genes. 
(a) Morphological appearance of spheres formed in the presence or absence of $10 \mu \mathrm{M}$ GANT61. HLE (upper) and HLF (lower) cells were cultured in an ultra-low-attachment culture plate for 10 days and photographed. Scale bar $=100 \mu \mathrm{m}$. (b) The diameter of the sphere was measured in five representative spheres of each group. (c) Quantitative PCR of stemness-related genes Oct4, Bmi1, CD44, ALDH1A1, and ALDH1A3 in HLE (left) and HLF (right) cells treated with $10 \mu \mathrm{M}$ of GANT61. GAPDH mRNA was used as an internal control. ${ }^{*} p<0.05,{ }^{* *} p<0.01$ vs. the vehicle control (DMSO).

\subsection{GANT61 Inhibits Cell Migration by Down-Regulating Snail1 Gene Expression}

To investigate the effect of GANT61 on cell migration ability, a wound healing assay was performed by scratching monolayer-cultured cells. To exclude the effect on cell proliferation, assays were performed under the condition of serum deprivation (1\% FBS or serum-free) using 5 and $10 \mu \mathrm{M}$ of GANT61. Twenty-four hours after scratching, GANT61-treated cells showed slower migration than control cells (Figure 5a). Quantification of cell migrating activity was carried out by measuring a closed area after $24 \mathrm{~h}$, and this revealed a significant and dose-dependent decrease in cell migrating activity in HLE and HLF cells (Figure 5b).

(a)

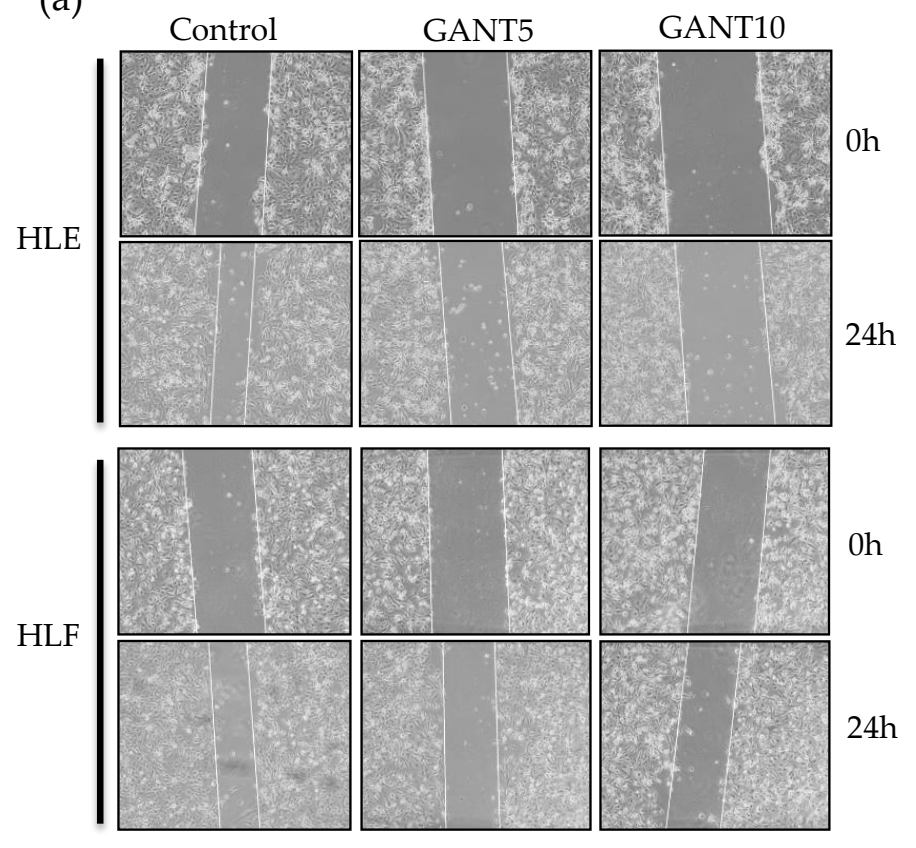

(b)
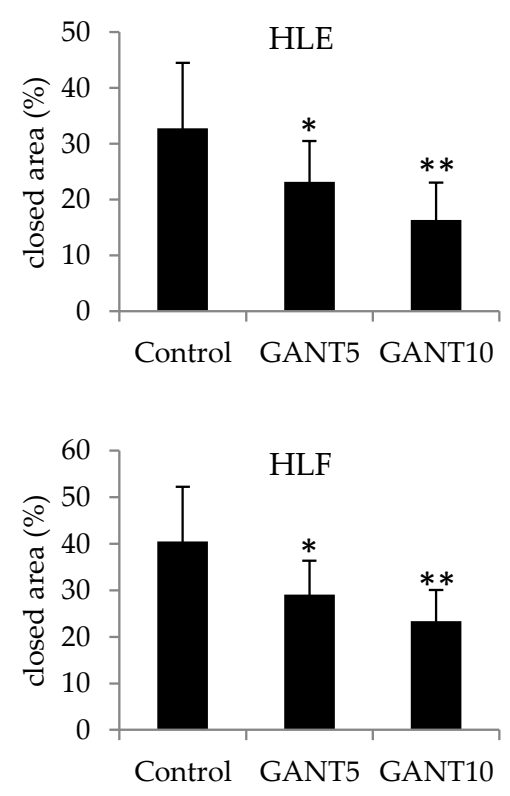

Figure 5. Wound healing assay of undifferentiated HCC cells treated with GANT61. (a) Scratched area of monolayer-cultured HLE (upper) and HLF (lower) cells treated with $5 \mu \mathrm{M}$ (GANT5) and $10 \mu \mathrm{M}$ (GANT10) of GANT61 under the serum-free condition. (b) A closed area was used to evaluate cell migration activity. ${ }^{*} p<0.05,{ }^{* *} p<0.01$ vs. the vehicle control (DMSO).

To confirm the inhibitory effect of GANT61 on cell migration, a transwell assay was performed. As shown in Figure 6, GANT61 lowered the migration of HLE cells in a dose-dependent manner (Figure 6a,b). Furthermore, an increase in the epithelial marker E-cadherin and a decrease in the mesenchymal marker vimentin were observed by Western blotting of the lysates extracted from GANT61-treated cells (Figure 6c). Quantitative PCR revealed that the mesenchymal transcription factor Snail1 was down-regulated by GANT61 treatment compared to the control (Figure 6d). Snail knockdown by small-interference RNA (siRNA) significantly reduced the migration ability of HLE cells (Figure 6e-g). Together, these data suggest that GANT61 inhibits cell migration by attenuating the mesenchymal phenotype of undifferentiated HCC cells. 
(a)
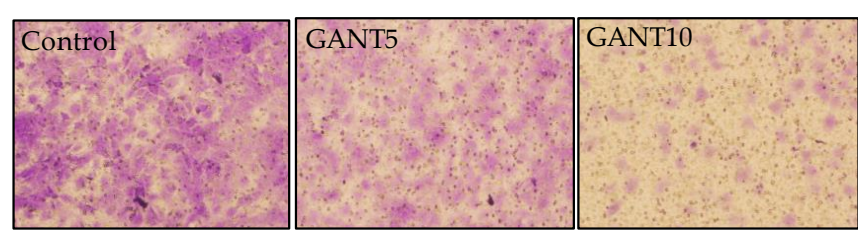

(c)
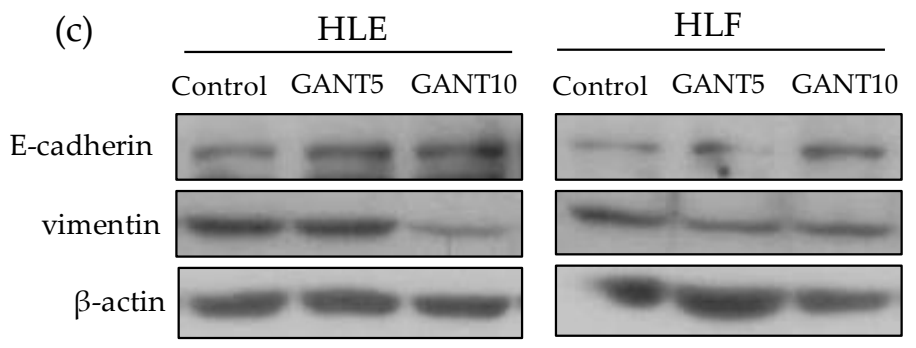

(b)

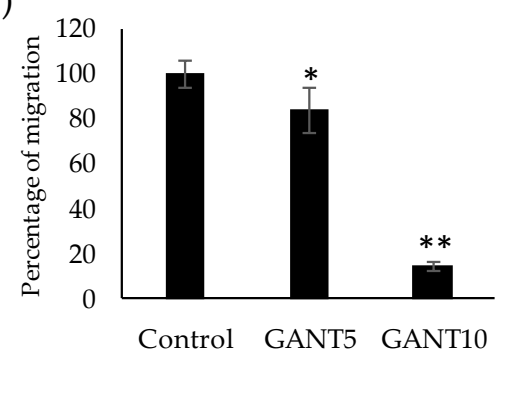

(d)

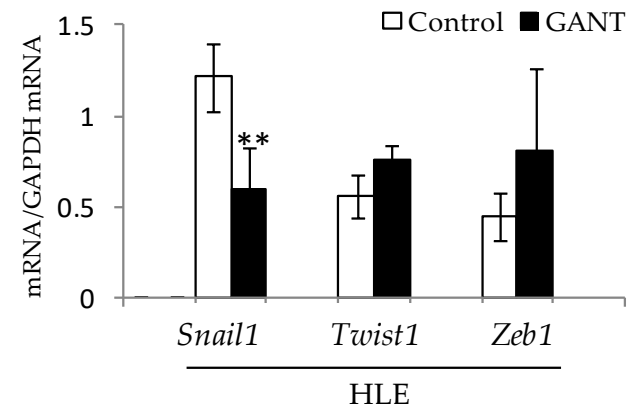

(e)

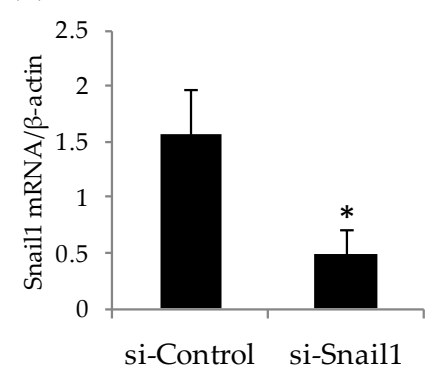

(f)

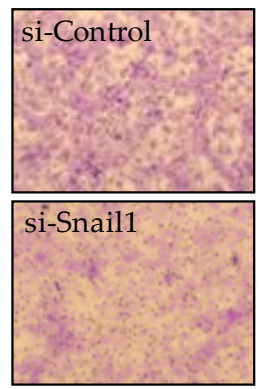

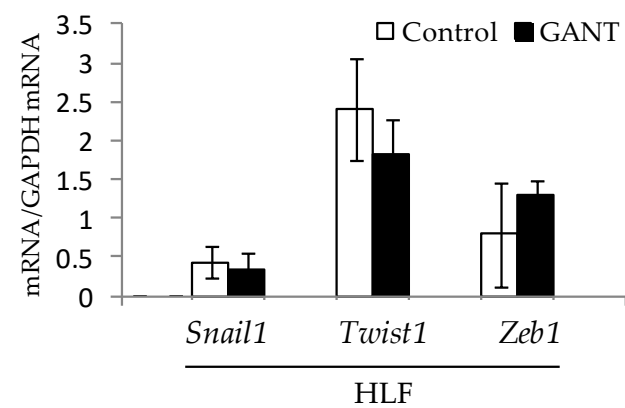

(g)

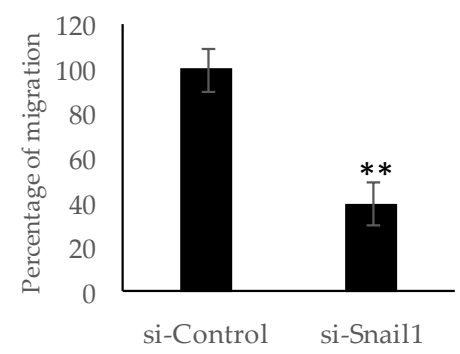

Figure 6. GANT61 inhibits cell migration by attenuating the mesenchymal phenotype of HLE cells. (a) Transwell migration assay of HLE cells treated with $5 \mu \mathrm{M}$ (GANT5) and $10 \mu \mathrm{M}$ (GANT10) of GANT61. Migrated cells were stained with crystal violet. (b) The migration activity of the cells is expressed as a percentage of the cell number compared with the control. (c) Western blotting of E-cadherin, vimentin, and $\beta$-actin as an loading control. (d) Quantitarive PCR analysis of EMT-related transcription factors Snail1, Twist1, and Zeb1 in HLE (left) and HLF (right) cells treated with $10 \mu \mathrm{M}$ GANT61. (e) Quantitative PCR analysis of Snail mRNA in HLE cells treated with control (si-Control) and Snail (si-Snail) siRNA. $(\mathbf{f}, \mathbf{g})$ Transwell migration assay of siRNA-treated HLE cells and their migration activity. ${ }^{*} p<0.05$, ** $p<0.01$ vs. vehicle control (DMSO).

\section{Discussion}

The HH-GLI signaling pathway was shown to play a critical function during development by regulating cellular proliferation and differentiation. In wound healing and regeneration processes, this pathway plays important roles in cellular differentiation, migration, and maintenance of tissue 
progenitor cells [17]. Therefore, aberrant activation of this signal in cancer cells leads to malignant transformations such as dedifferentiation, acquisition of stemness features, and migration potency. In this study, we investigated the biological significance of GLI-mediated signaling in undifferentiated HCC cell lines using the GLI specific inhibitor GANT61. We found preferential expression of GLI mRNAs in undifferentiated HCC cell lines that showed the mesenchymal phenotype. Consistent with previous reports, HLE and HLF were characterized by increased mesenchymal markers and the loss of epithelial and hepatic markers [18,19]. Among GLI genes, GLI2 and repressor GLI3 were specifically expressed in undifferentiated HCC cells. Generally, GLI1 acts mainly transcriptional activation in cancer cells, whereas GLI2 requires $\mathrm{N}$-terminal processing for full activation [20,21]. GLI2 is reported to induce genomic instability by inhibiting apoptosis, which is responsible for eliminating transformed aberrant cells [22]. These reports and our data suggest that GLI2 expression, in addition to that of GLI1, contributes to the promotion of cell dedifferentiation and confers HCC cells with mesenchymal phenotypes.

To determine the biological impact of GANT61 in undifferentiated HCC cells, we investigated the cell proliferation and sensitivity to cell death induced by anticancer drugs in GANT61-treated HLE and HLF cells. Although we observed a positive antitumor effect of GANT61 on cell viability, the impact seemed to be relatively weak compared with that observed in other type of cancers. For instance, previous studies reported that only $10 \%-40 \%$ of cells remained viable after treatment with $10-20 \mu \mathrm{M}$ of GANT61 in lung, melanoma, prostate, glioma, and breast cancer cells [23-29]. These reports and our observation allow us to hypothesize that the GANT61 target may be a small subpopulation in HLE and HLF cells. In combination treatment, there was little enhancing effect on drug sensitivity when GANT61was used with low concentrations of mitomycin C and 5-FU; however, a significant decrease in cell viability was observed when GANT61 was used in combination with relatively high concentrations of these drugs. This result suggests that a small population of drug-resistant cells were targeted by GANT61. Previous reports showed that HH-signal inhibitors, such as the SMO inhibitor and direct GLI inhibitors, have antitumor effects by targeting CSC-like cell populations [30,31]. Therefore, it is reasonable to hypothesize that a small subpopulation of CSC-like cells which depends on GLI signaling would be a target of GANT61.

Interestingly, we observed no enhancement of cell death in combination with sorafenib, a multi-kinase inhibitor targeting the serine-threonine kinases Raf-1 and B-Raf and the receptor tyrosine kinase activity of VEGFRs and PDGFR- $\beta$. A possible mechanism underlying the drug-specific response might be explained by the observation that sorafenib down-regulated the expression of GLI genes. GLI-dependent transcription is known to be activated in an HH ligand-independent manner, namely non-canonical $\mathrm{HH}$ signaling, which includes oncogenic intracellular signaling pathways such as PI3-AKT, TGF $\beta$-smad, and Ras-Raf-MEK-ERK [16]. Therefore, in undifferentiated HCC, the GLI-mediated HH pathway is suggested to be regulated, at least in part, by the Ras-Raf-MEK-ERK pathway.

Increasing evidence is suggesting that the acquisition of stemness features in tumor cells is correlated with poor clinical outcomes in various type of cancers. These malignantly transformed cells often show enhanced resistance to chemotherapy, anchorage-independent survival, and migration potency that causes postoperative recurrence and metastasis. As well as well-known oncogenic intracellular signaling components such as Wnt $/ \beta$-catenin, Notch, TGF- $\beta$, and AKT, HH-GLI signaling was described as an important pathway for maintaining the stemness of undifferentiated and/or immature cells [3]. In normal liver development, HH-GLI signaling contributes to the proliferation of immature progenitor cells, and its activation progressively decreases during liver epithelial cell maturation [32]. HH-GLI signal activation re-increases when the tissue regenerates after injury to promote the proliferation of liver progenitor cells. Therefore, HH-GLI signaling is considered to promote dedifferentiation and confer cancer cells to have stemness features, making it a promising target for cancer therapy. In this study, we observed significant decreases in sphere forming activity and cell migration by GANT61. Gene expression analysis revealed that GLI inhibition significantly 
down-regulated Oct4 in HLE cells and in HLF cells, showing a tendency to reduce the expression of stemness-related genes (Oct4, Bmi1, and CD44) which play important roles in anchorage-independent cell growth. We also observed significant down-regulation of ALDH mRNA expression by GANT61. ALDH was shown to play an important role in self-renewal in various cancers including HCC and was reported to be regulated by GLI signaling in melanomas [33,34]. Therefore, these stemness-related genes may be crucial targets of GLI signaling in undifferentiated HCC. In the sphere assay, the rate of sphere formation was found to be about $1 \%$ for the cells seeded in a low-attachment dish (data not shown). In future research, identification and isolation of a subpopulation that has sphere forming activity is required to assess the critical effect of GANT61.

A significant decrease in cell motility by GANT61 was confirmed in a wound healing assay and transwell assay. These assays and the Western blot analysis suggest that the mesenchymal features of undifferentiated HCC cells are attenuated by GANT61 treatment. In terms of the molecular mechanism, Snail1, a pivotal transcriptional regulator for migration potency, was found to be down-regulated in HLE cells. Although Snail1 is known as a key regulator of EMT, the impact of GANT61 on mRNA expression differed among two cell lines (Figure 6d). Moreover, GANT61 treatment was associated with greater inhibition of migration activity than Snail knockdown, despite the weaker down-regulation effect of Snail mRNA compared with that of si-Snail (Figure 6b,g). Together, these results suggest that other genes, in addition to Snail, responsible for cell migration may be affected by GANT61. A thorough study of target genes including coding and non-coding genes should be required to understand the cell-type-specific action of GAN61. An anti-migrative effect was related to HH signaling inhibition by the SMO inhibitors NVP-LDE-225 and GDC-0449, both of which were approved for clinical application [35-37]. However, these drugs may be ineffective for the non-canonical GLI-activation pathway observed in this study. In addition to the SMO inhibitor, the use of a direct inhibitor of GLI and/or inhibitors of signaling pathways involved in the non-canonical HH-GLI pathway may be required for effective treatment.

As depicted in Figure 7, the results of our study demonstrate that the HH-GLI signal inhibitor GANT61 has an antitumor effect on the malignant character of undifferentiated HCC cells with the mesenchymal phenotype. Direct inhibition of GLI was suggested to be beneficial in the case of dedifferentiated HCC which is involved in non-canonical HH-GLI signaling.

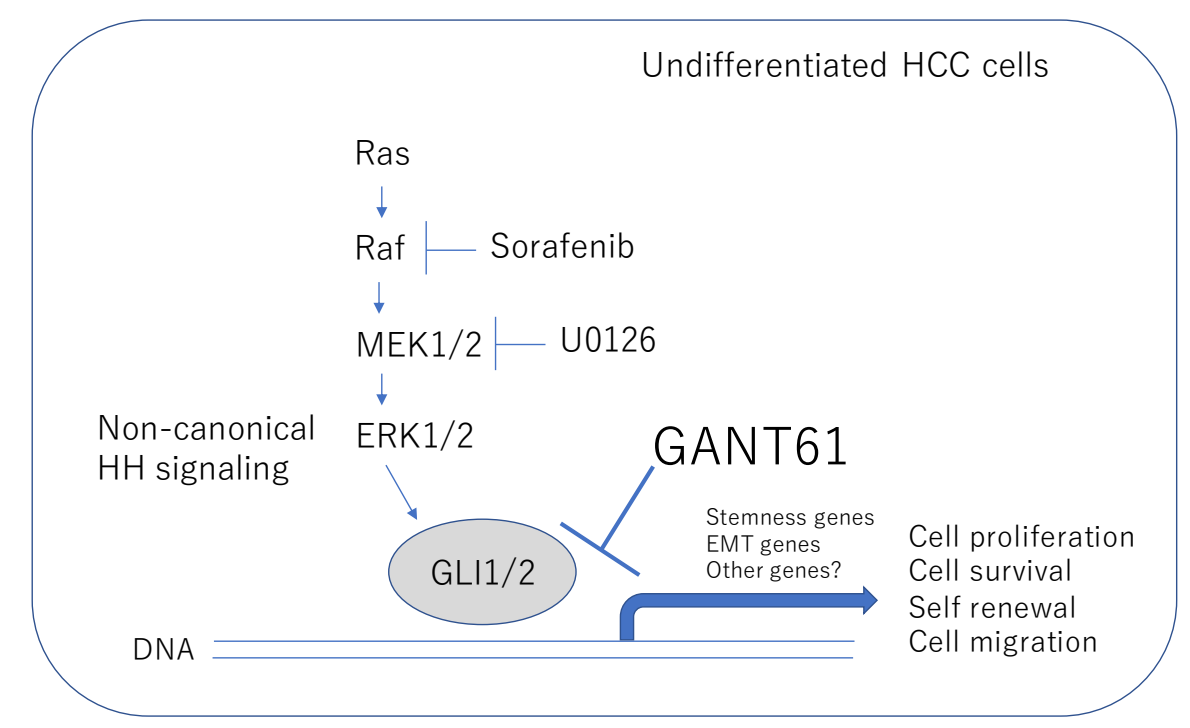

Figure 7. Schematic view of the antitumor effect of GANT61 in undifferentiated HCC cells. GANT61 inhibits the malignant behavior of undifferentiated HCC cells by targeting non-canonical GLI signaling. Arrows denote activation and T-shaped lines indicate inhibition. 


\section{Materials and Methods}

\subsection{Cell Culture and Reagents}

Hepatoma cell lines (HepG2, HuH1, HuH7, HLE and HLF) were purchased from RIKEN BRC. Cells were maintained in DMEM containing 10\% FBS, $4.5 \mathrm{mg} / \mathrm{mL}$ glucose, and $2 \mathrm{mM}$ L-glutamine in a humidified incubator at $37^{\circ} \mathrm{C}$ and 5\% CO . GANT61 (Santa Cruz Biotechnology; sc-202630) and sorafenib (ChemScene LLC; Monmouth Junction, NJ, USA) were dissolved in DMSO at a concentration of $10 \mathrm{mM}$ and stored in a freezer at $-20^{\circ} \mathrm{C}$.

\subsection{Cell Viability Assays}

Cell viability was determined by the WST assay using the Cell Counting Kit-8 (Dojin Chemical Co., Ltd.; Kumamoto, Japan). Briefly, cells were cultured in a 96-well plate and subjected to the experiment. To determine cell viability, a 10\% volume of CCK-8 reagent was added to each well, and the plate was incubated for $1 \mathrm{~h}$ at $37^{\circ} \mathrm{C}$. Cell viability was calculated by measuring the absorbance at a wavelength of $450 \mathrm{~nm}$. For cell proliferating assays, cells were seeded at $3 \times 10^{3}$ cells/well and measured at 24, 48, and $72 \mathrm{~h}$ after the treatment. The relative number of viable cells was calculated from OD450 to create proliferation curves.

\subsection{Gene Expression Analysis}

Total RNA was isolated using the TRIZOL reagent in accordance with the manufacturer's instructions (Invitrogen; Carlsbad, CA, USA). One microgram of RNA was used to synthesize cDNA using GeneAce Reverse Transcriptase (Nippon Gene; Tokyo, Japan). A gene expression analysis was performed using PowerUp SYBR Green Master Mix (Thermo Fisher Scientific; Waltham, MA, USA) and the ABI qPCR system (Thermo Fisher Scientific). The primers used in this study are listed in Supplementary Table S1.

\subsection{Sphere Forming Assay}

A sphere assay was performed by seeding HLE and HLF cells in a 12-well low-attachment plate. Cells were treated with DMSO or $10 \mu \mathrm{M}$ of GANT61 and incubated for 10 days. Half of the medium was changed every three days. Formed spheres were photographed and their diameters were measured.

\subsection{Cell Migration Assays}

Cells were grown at $90 \%-100 \%$ confluency and subjected to the experiment. Monolayer cells were scratched using microtips and photographed at day 0. Cells were treated with serum-free DMEM containing DMSO or $10 \mu \mathrm{M}$ of GANT61 for $24 \mathrm{~h}$ and photographed at day 1 . A closed area was used to evaluate cell migration activity.

\subsection{Transwell Migration Assays}

HLE cells were pre-treated with DMSO or GANT61 (5 and $10 \mu \mathrm{M})$ in a serum-starved condition for $24 \mathrm{~h}$. A total of $5 \times 10^{4}$ cells were seeded into the upper chambers of transwell inserts with $8 \mu \mathrm{M}$ pores (Corning Inc.; Corning, NY, USA) with DMEM but without serum. The bottom well contained $10 \%$ FBS DMEM to induce cell migration. After $24 \mathrm{~h}$, the cells that migrated to the underside of the membrane were fixed and stained with crystal violet containing $10 \%$ methanol and counted to quantify the migration ability. A knockdown experiment was performed by transfection of Stealth RNAi Pre-Designed siRNAs (Thermo Fisher Scientific) for Snail mRNA and a negative control for $24 \mathrm{~h}$. Cells treated with siRNA were subjected to the transwell assay as described above. 


\subsection{Western Blot Analysis}

Cell lysates were prepared from the cells subjected to the experiment by using Cell Lysis Buffer (Cell Signaling Technology; Danvers, MA, USA) and stored in a deep freezer until use. The protein concentration was determined by the Pierce ${ }^{\mathrm{TM}}$ BCA Protein Assay Kit (Thermo Fisher Scientific). Equal amounts of the protein sample were separated on a SDS-PAGE and immunoblotted with antibodies against human E-cadherin (Santa Cruz Biotechnology), vimentin (Santa Cruz Biotechnology), MEK1 (Novus Biologicals, Centennial CO, USA), phospho-MEK1/2 (Ser2017/221) (Cell Signaling Technology), phospho-ERK1/2 (Thr202/Tyr204) (Cell Signaling Technology), and beta-actin (Cell Signaling Technology). HRP-conjugated secondary antibodies were used to detect primary antibodies and were visualized with ECL detection reagent (GE Healthcare UK; Buckinghamshire, UK) and a light-sensitive $\mathrm{X}$-ray film (Fuji film; Tokyo, Japan).

\subsection{Statistical Analysis}

Statistical comparisons for in vitro experiments were performed using Student's $t$-tests. $p<0.05$ was considered statistically significant.

Supplementary Materials: Supplementary materials can be found at http://www.mdpi.com/1422-0067/21/9/3126/ s1.

Author Contributions: K.H. and K.K. designed the experiments. K.H. performed the cell viability assay and sphere forming assay. R.O. performed cell migration assays. K.N. performed the siRNA experiment. K.K. performed the gene expression analysis and Western blotting. K.K. wrote the manuscript and acquired funding. All authors have read and agreed to the published version of the manuscript.

Funding: This research was supported by Grants-in-Aid for Scientific Research (KAKENHI, 18K07214), Japan Society for the Promotion of Science (JSPS) and The Okayama Medical Foundation.

Conflicts of Interest: The authors declare no conflict of interest.

\section{Abbreviations}

$\begin{array}{ll}\text { HCC } & \text { Hepatocellular carcinoma } \\ \text { HH } & \text { Hedgehog } \\ \text { GLI } & \text { Glioma-associated homologue } \\ \text { SMO } & \text { Smoothened } \\ \text { EMT } & \text { Epithelial-mesenchymal transition } \\ \text { RT-PCR } & \text { Reverse Transcription-Polymerase Chain Reaction } \\ \text { HNF4 } \alpha & \text { Hepatocyte nuclear factor } 4 \alpha \\ \text { DMSO } & \text { Dimethyl sulfoxide } \\ \text { siRNA } & \text { Small-interference RNA } \\ \text { ALDH } & \text { Aldehyde dehydrogenase } \\ \text { CSC } & \text { Cancer Stem Cells }\end{array}$

\section{References}

1. Yang, J.D.; Hainaut, P.; Gores, G.J.; Amadou, A.; Plymoth, A.; Roberts, L.R. A global view of hepatocellular carcinoma: Trends, risk, prevention and management. Nat. Rev. Gastroenterol. Hepatol. 2019, 16, 589-604. [CrossRef] [PubMed]

2. Gupta, P.B.; Pastushenko, I.; Skibinski, A.; Blanpain, C.; Kuperwasser, C. Phenotypic Plasticity: Driver of Cancer Initiation, Progression, and Therapy Resistance. Cell Stem Cell 2019, 24, 65-78. [CrossRef] [PubMed]

3. Flores-Téllez, T.N.; Villa-Treviño, S.; Piña-Vázquez, C. Road to stemness in hepatocellular carcinoma. World J. Gastroenterol. 2017, 23, 6750-6776. [CrossRef] [PubMed]

4. Della Corte, C.M.; Viscardi, G.; Papaccio, F.; Esposito, G.; Martini, G.; Ciardiello, D.; Martinelli, E.; Ciardiello, F.; Morgillo, F. Implication of the Hedgehog pathway in hepatocellular carcinoma. World J. Gastroenterol. 2017, 23, 4330-4340. [CrossRef] 
5. Gonnissen, A.; Isebaert, S.; Haustermans, K. Targeting the Hedgehog signaling pathway in cancer: Beyond Smoothened. Oncotarget 2015, 6, 13899-13913. [CrossRef]

6. Rohatgi, R.; Milenkovic, L.; Scott, M.P. Patched1 regulates hedgehog signaling at the primary cilium. Science 2007, 317, 372-376. [CrossRef]

7. Kovacs, J.J.; Whalen, E.J.; Liu, R.; Xiao, K.; Kim, J.; Chen, M.; Wang, J.; Chen, W.; Lefkowitz, R.J. Beta-arrestin-mediated localization of smoothened to the primary cilium. Science 2008, 320, 1777-1781. [CrossRef]

8. Katoh, Y.; Katoh, M. Hedgehog target genes: Mechanisms of carcinogenesis induced by aberrant hedgehog signaling activation. Curr. Mol. Med. 2009, 9, 873-886. [CrossRef]

9. Gupta, S.; Takebe, N.; Lorusso, P. Targeting the Hedgehog pathway in cancer. Ther. Adv. Med. Oncol. 2010, 2, 237-250. [CrossRef]

10. Sekulic, A.; Migden, M.R.; Oro, A.E.; Dirix, L.; Lewis, K.D.; Hainsworth, J.D.; Solomon, J.A.; Yoo, S.; Arron, S.T.; Friedlander, P.A.; et al. Efficacy and safety of vismodegib in advanced basal-cell carcinoma. $N$. Engl. J. Med. 2012, 366, 2171-2179. [CrossRef]

11. Rodon, J.; Tawbi, H.A.; Thomas, A.L.; Stoller, R.G.; Turtschi, C.P.; Baselga, J.; Sarantopoulos, J.; Mahalingam, D.; Shou, Y.; Moles, M.A.; et al. A phase I, multicenter, open-label, first-in-human, dose-escalation study of the oral smoothened inhibitor Sonidegib (LDE225) in patients with advanced solid tumors. Clin. Cancer Res. 2014, 20, 1900-1909. [CrossRef] [PubMed]

12. Jimeno, A.; Weiss, G.J.; Miller, W.H., Jr.; Gettinger, S.; Eigl, B.J.; Chang, A.L.; Dunbar, J.; Devens, S.; Faia, K.; Skliris, G.; et al. Phase I study of the Hedgehog pathway inhibitor IPI-926 in adult patients with solid tumors. Clin. Cancer Res. 2013, 19, 2766-2774. [CrossRef] [PubMed]

13. Chang, A.L.; Oro, A.E. Initial assessment of tumor regrowth after vismodegib in advanced Basal cell carcinoma. Arch. Dermatol. 2012, 148, 1324-1325. [CrossRef]

14. Yauch, R.L.; Dijkgraaf, G.J.; Alicke, B.; Januario, T.; Ahn, C.P.; Holcomb, T.; Pujara, K.; Stinson, J.; Callahan, C.A.; Tang, T.; et al. Smoothened mutation confers resistance to a Hedgehog pathway inhibitor in medulloblastoma. Science 2009, 326, 572-574. [CrossRef] [PubMed]

15. Kool, M.; Jones, D.T.; Jäger, N.; Northcott, P.A.; Pugh, T.J.; Hovestadt, V.; Piro, R.M.; Esparza, L.A.; Markant, S.L.; Remke, M.; et al. Genome sequencing of SHH medulloblastoma predicts genotype-related response to smoothened inhibition. Cancer Cell 2014, 25, 393-405. [CrossRef] [PubMed]

16. Pietrobono, S.; Gagliardi, S.; Stecca, B. Non-canonical Hedgehog Signaling Pathway in Cancer: Activation of GLI Transcription Factors Beyond Smoothened. Front. Genet. 2019, 10, 556. [CrossRef]

17. Singh, B.N.; Koyano-Nakagawa, N.; Donaldson, A.; Weaver, C.V.; Garry, M.G.; Garry, D.J. Hedgehog Signaling during Appendage Development and Regeneration. Genes 2015, 6, 417-435. [CrossRef]

18. Okabe, H.; Ishimoto, T.; Mima, K.; Nakagawa, S.; Hayashi, H.; Kuroki, H.; Imai, K.; Nitta, H.; Saito, S.; Hashimoto, D.; et al. CD44s signals the acquisition of the mesenchymal phenotype required for anchorage-independent cell survival in hepatocellular carcinoma. Br. J. Cancer 2014, 110, 958-966. [CrossRef]

19. Mima, K.; Okabe, H.; Ishimoto, T.; Hayashi, H.; Nakagawa, S.; Kuroki, H.; Watanabe, M.; Beppu, T.; Tamada, M.; Nagano, O.; et al. CD44s regulates the TGF-beta-mediated mesenchymal phenotype and is associated with poor prognosis in patients with hepatocellular carcinoma. Cancer Res. 2012, 72, 3414-3423. [CrossRef]

20. Carpenter, R.L.; Lo, H.W. Hedgehog pathway and GLI1 isoforms in human cancer. Discov. Med. 2012, 13, 105-113.

21. Speek, M.; Njunkova, O.; Pata, I.; Valdre, E.; Kogerman, P. A potential role of alternative splicing in the regulation of the transcriptional activity of human GLI2 in gonadal tissues. BMC Mol. Biol. 2006, 7, 13. [CrossRef] [PubMed]

22. Pantazi, E.; Gemenetzidis, E.; Trigiante, G.; Warnes, G.; Shan, L.; Mao, X.; Ikram, M.; The, M.T.; Lu, Y.J.; Philpott, M.P. GLI2 induces genomic instability in human keratinocytes by inhibiting apoptosis. Cell Death Dis. 2014, 5, e1028. [CrossRef] [PubMed]

23. Huang, L.; Walter, V.; Hayes, D.N.; Onaitis, M. Hedgehog-GLI signaling inhibition suppresses tumor growth in squamous lung cancer. Clin. Cancer Res. 2014, 20, 1566-1575. [CrossRef] [PubMed]

24. Vlčková, K.; Réda, J.; Ondrušová, L.; Krayem, M.; Ghanem, G.; Vachtenheim, J. GLI inhibitor GANT61 kills melanoma cells and acts in synergy with obatoclax. Int. J. Oncol. 2016, 49, 953-960. [CrossRef] [PubMed] 
25. Tong, W.; Qiu, L.; Qi, M.; Liu, J.; Hu, K.; Lin, W.; Huang, Y.; Fu, J. GANT-61 and GDC-0449 induce apoptosis of prostate cancer stem cells through a GLI-dependent mechanism. J. Cell Biochem. 2018, 119, 3641-3652. [CrossRef]

26. Li, J.; Cai, J.; Zhao, S.; Yao, K.; Sun, Y.; Li, Y.; Chen, L.; Li, R.; Zhai, X.; Zhang, J.; et al. GANT61, a GLI inhibitor, sensitizes glioma cells to the temozolomide treatment. J. Exp. Clin. Cancer Res. 2016, 35, 184. [CrossRef]

27. Riaz, S.K.; Ke, Y.; Wang, F.; Kayani, M.A.; Malik, M.F.A. Influence of SHH/GLI1 axis on EMT mediated migration and invasion of breast cancer cells. Sci. Rep. 2019, 9, 6620. [CrossRef]

28. Riaz, S.K.; Khan, J.S.; Shah, S.T.A.; Wang, F.; Ye, L.; Jiang, W.G.; Malik, M.F.A. Involvement of hedgehog pathway in early onset, aggressive molecular subtypes and metastatic potential of breast cancer. Cell Commun. Signal. 2018, 16, 3. [CrossRef]

29. Réda, J.; Vachtenheim, J.; Vlčková, K.; Horák, P.; Vachtenheim, J., Jr.; Ondrušová, L. Widespread Expression of Hedgehog Pathway Components in a Large Panel of Human Tumor Cells and Inhibition of Tumor Growth by GANT61: Implications for Cancer Therapy. Int. J. Mol. Sci. 2018, 19, 2682. [CrossRef]

30. Nanta, R.; Shrivastava, A.; Sharma, J.; Shankar, S.; Srivastava, R.K. Inhibition of sonic hedgehog and $\mathrm{PI} 3 \mathrm{~K} / \mathrm{Akt} / \mathrm{mTOR}$ pathways cooperate in suppressing survival, self-renewal and tumorigenic potential of glioblastoma-initiating cells. Mol. Cell Biochem. 2019, 454, 11-23. [CrossRef]

31. Miyazaki, Y.; Matsubara, S.; Ding, Q.; Tsukasa, K.; Yoshimitsu, M.; Kosai, K.; Takao, S. Efficient elimination of pancreatic cancer stem cells by hedgehog/GLI inhibitor GANT61 in combination with mTOR inhibition. Mol. Cancer 2016, 15, 49. [CrossRef] [PubMed]

32. Sicklick, J.K.; Li, Y.X.; Melhem, A.; Schmelzer, E.; Zdanowicz, M.; Huang, J.; Caballero, M.; Fair, J.H.; Ludlow, J.W.; McClelland, R.E.; et al. Hedgehog signaling maintains resident hepatic progenitors throughout life. Am. J. Physiol. Gastrointest. Liver Physiol. 2006, 290, G859-G870. [CrossRef] [PubMed]

33. Ma, S.; Chan, K.W.; Lee, T.K.; Tang, K.H.; Wo, J.Y.; Zheng, B.J.; Guan, X.Y. Aldehyde dehydrogenase discriminates the CD133 liver cancer stem cell populations. Mol Cancer Res. 2008, 6, 1146-1153. [CrossRef] [PubMed]

34. Santini, R.; Vinci, M.C.; Pandolfi, S.; Penachioni, J.Y.; Montagnani, V.; Olivito, B.; Gattai, R.; Pimpinelli, N.; Gerlini, G.; Borgognoni, L.; et al. Hedgehog-GLI signaling drives self-renewal and tumorigenicity of human melanoma-initiating cells. Stem Cells 2012, 30, 1808-1818. [CrossRef]

35. Nanta, R.; Kumar, D.; Meeker, D.; Rodova, M.; Van Veldhuizen, P.J.; Shankar, S.; Srivastava, R.K. NVP-LDE-225 (Erismodegib) inhibits epithelial-mesenchymal transition and human prostate cancer stem cell growth in NOD/SCID IL2R $\gamma$ null mice by regulating Bmi-1 and microRNA-128. Oncogenesis 2013, 2, e42. [CrossRef]

36. Tu, Y.; Niu, M.; Xie, P.; Yue, C.; Liu, N.; Qi, Z.; Gao, S.; Liu, H.; Shi, Q.; Yu, R.; et al. Smoothened is a poor prognosis factor and a potential therapeutic target in glioma. Sci. Rep. 2017, 7, 42630. [CrossRef]

37. Magistri, P.; Battistelli, C.; Strippoli, R.; Petrucciani, N.; Pellinen, T.; Rossi, L.; Mangogna, L.; Aurello, P.; D'Angelo, F.; Tripodi, M.; et al. SMO Inhibition Modulates Cellular Plasticity and Invasiveness in Colorectal Cancer. Front. Pharmacol. 2018, 8, 956. [CrossRef]

(C) 2020 by the authors. Licensee MDPI, Basel, Switzerland. This article is an open access article distributed under the terms and conditions of the Creative Commons Attribution (CC BY) license (http://creativecommons.org/licenses/by/4.0/). 